\title{
Transportation Storage Logistics (TSL) Model Data Management Manual
}

Fuel Cycle Research \& Development

Prepared for

U.S. Department of Energy Campaign or Program Ingrid Busch and Rob Howard Oak Ridge National Laboratory

October 2012 FCRD-NFST-2012-000426 


\section{DISCLAIMER}

This information was prepared as an account of work sponsored by an agency of the U.S. Government. Neither the U.S. Government nor any agency thereof, nor any of their employees, makes any warranty,

expressed or implied, or assumes any legal liability or responsibility for the accuracy, completeness, or usefulness, of any information, apparatus, product, or process disclosed, or represents that its use would not infringe privately owned rights. References herein to any specific commercial product, process, or service by trade name, trade mark, manufacturer, or otherwise, does not necessarily constitute or imply its endorsement, recommendation, or favoring by the U.S. Government or any agency thereof. The views and opinions of authors expressed herein do not necessarily state or reflect those of the U.S. Government or any agency thereof. 
Transportation Storage Logistics (TSL) Model Data Management Manual October 2012 


\section{SUMMARY}

The Transportation Storage Logistics (TSL) Model Data Management Manual describes the SQL Server database that is used by the TSL model to house reference data as well as the scenario data. The manual is a reference document, subject to change as the model evolves. 
Transportation Storage Logistics (TSL) Model Data Management Manual October 2012 


\section{CONTENTS}

1. Canister

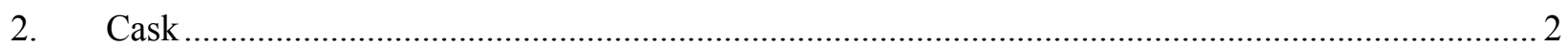

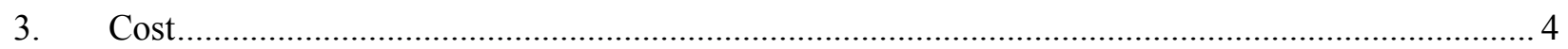

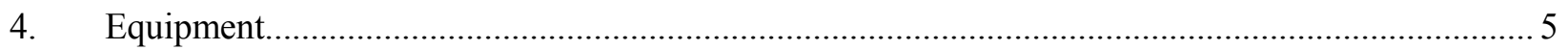

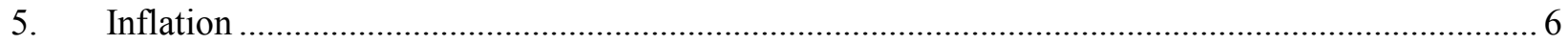

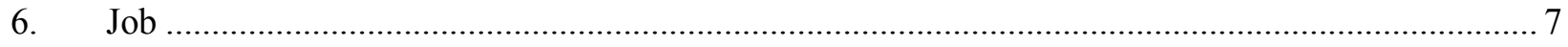

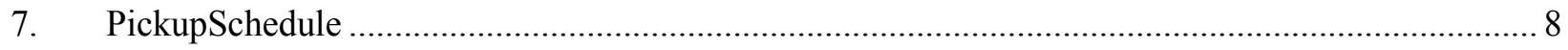

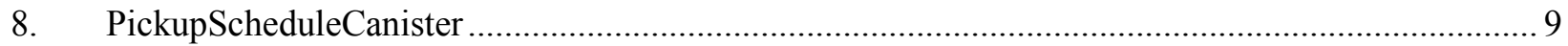

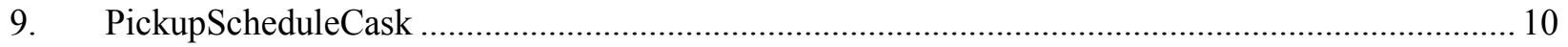

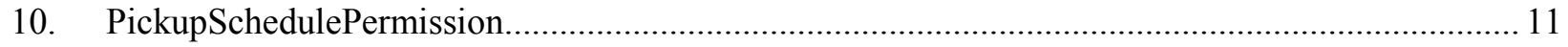

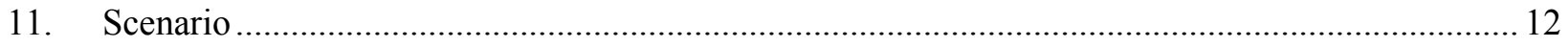

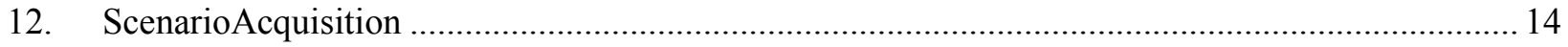

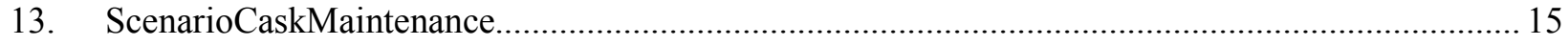

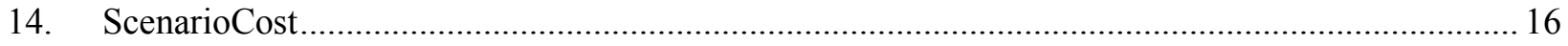

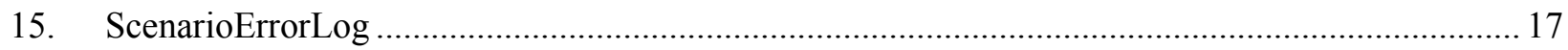

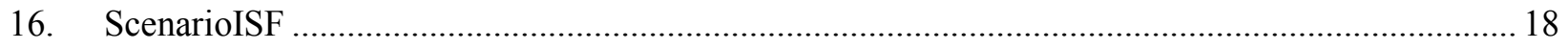

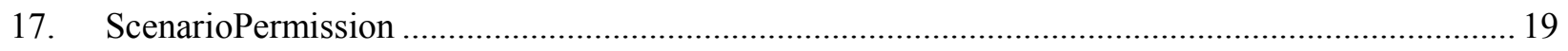

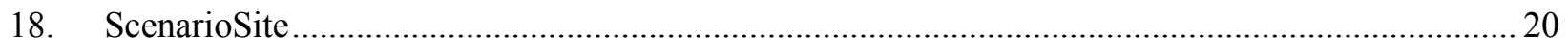

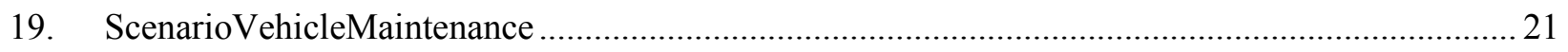

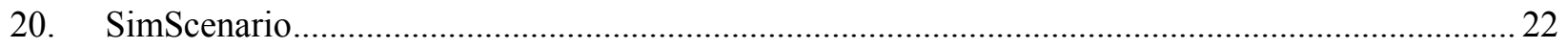

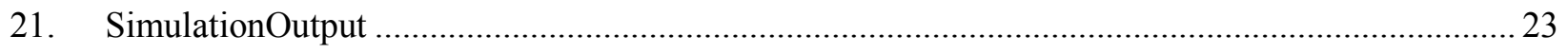

22. SimulationTransferTime

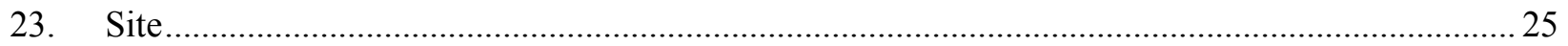

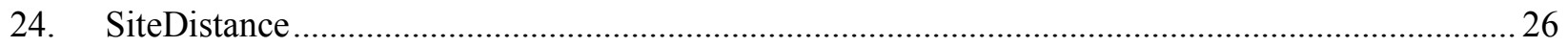

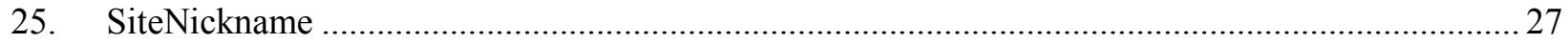




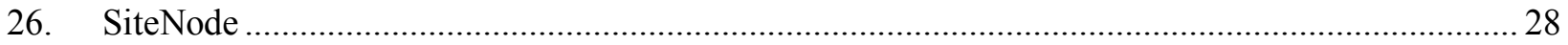

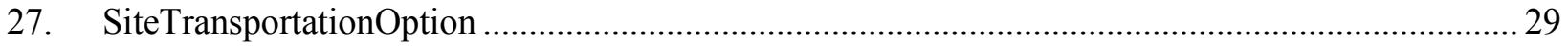

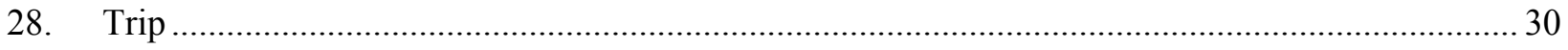

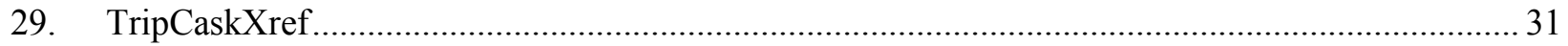

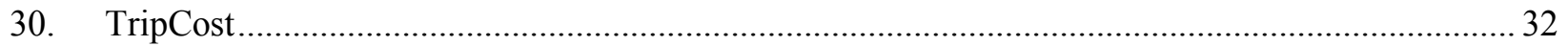

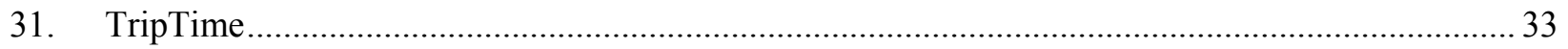

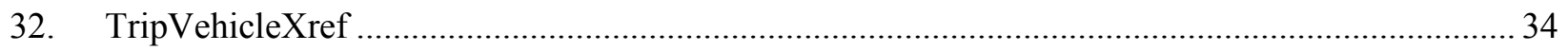

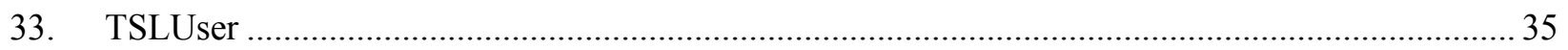

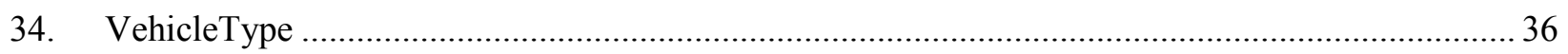

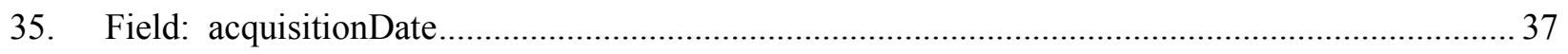

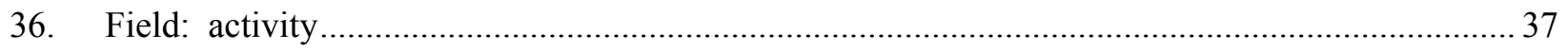

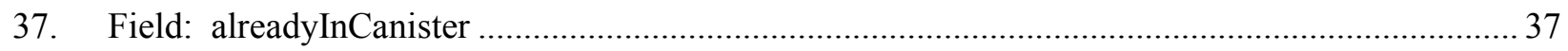

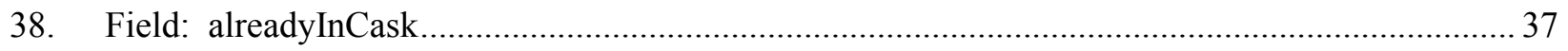

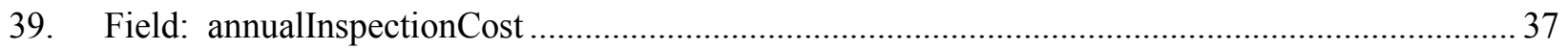

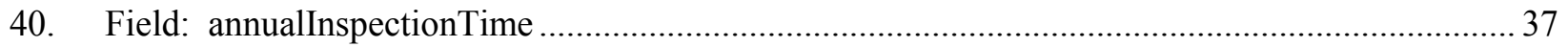

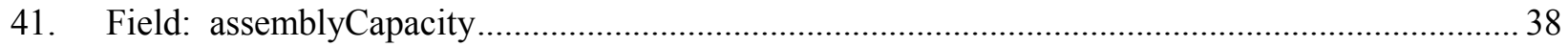

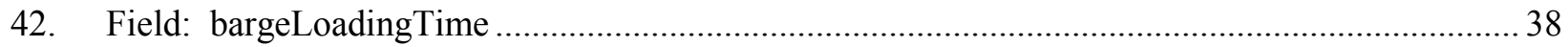

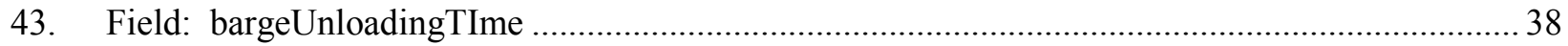

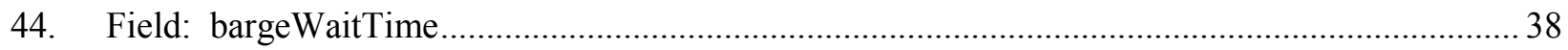

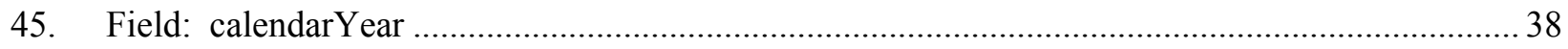

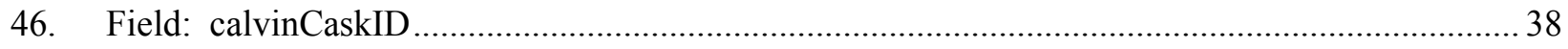

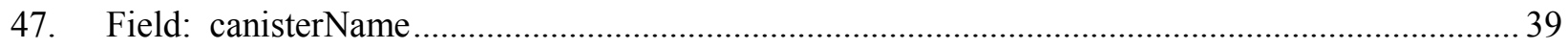

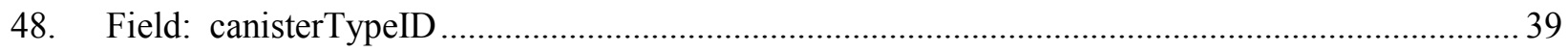

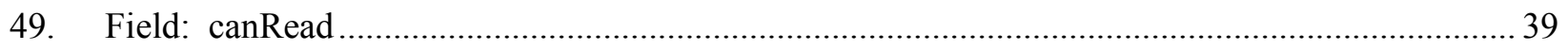

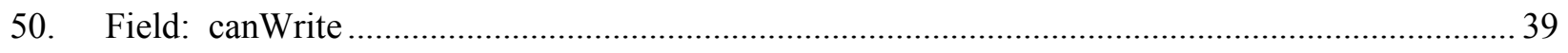

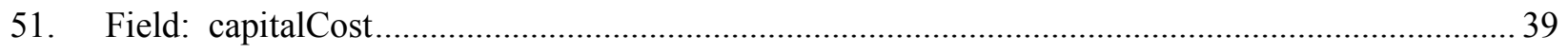




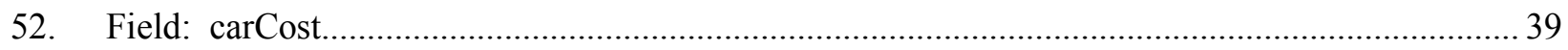

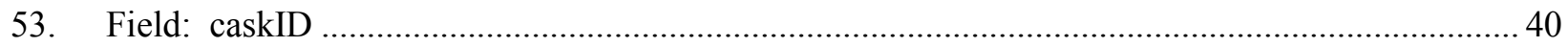

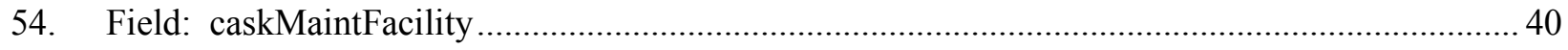

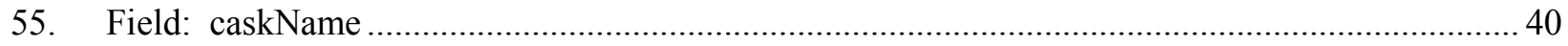

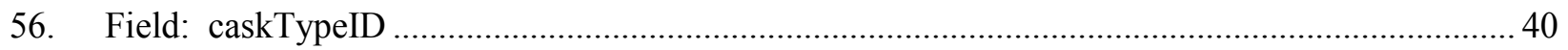

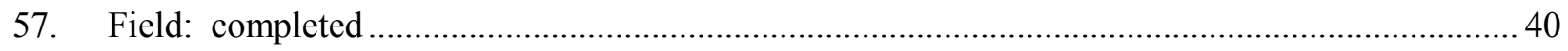

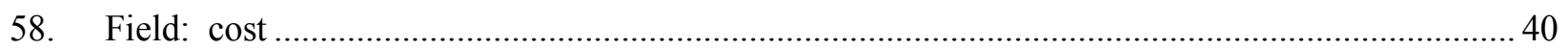

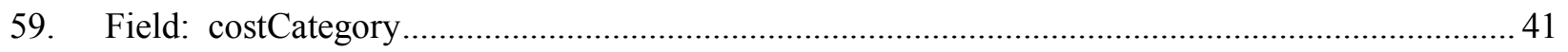

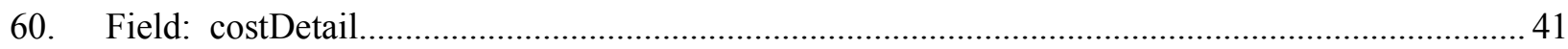

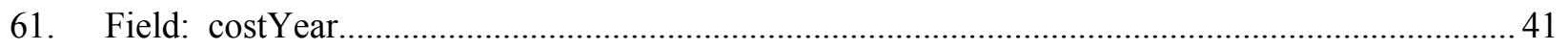

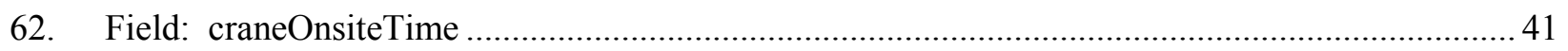

63. Field: craneOperationTime

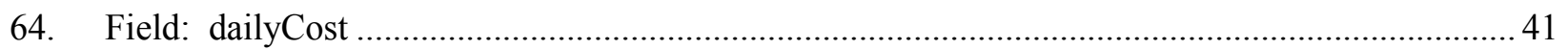

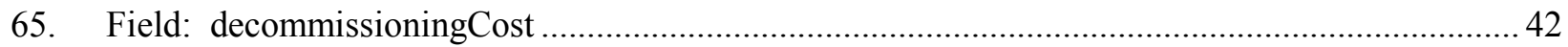

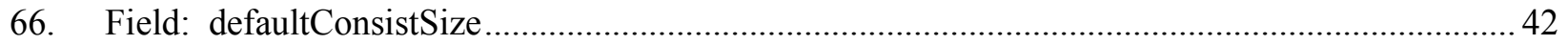

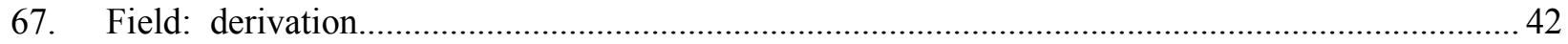

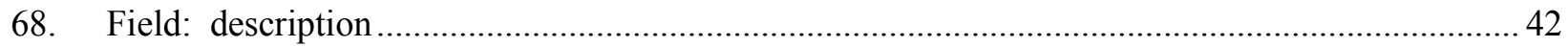

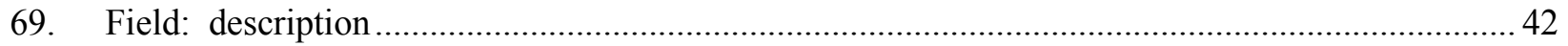

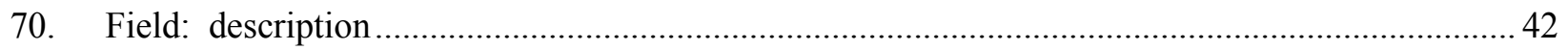

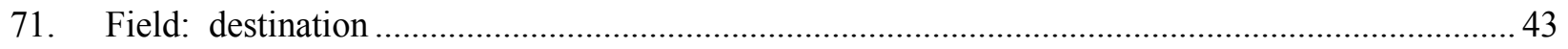

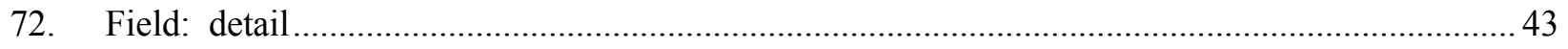

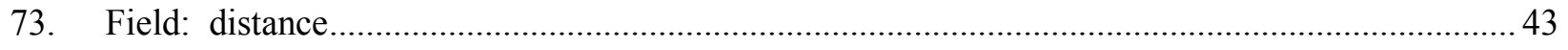

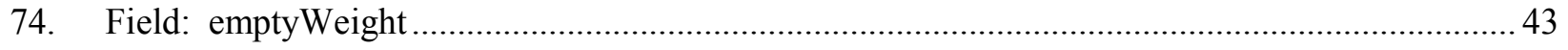

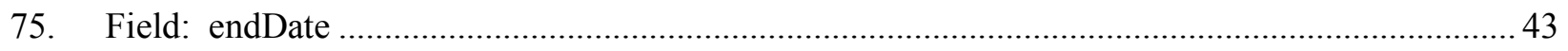

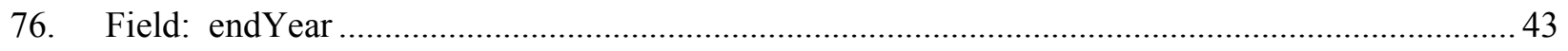

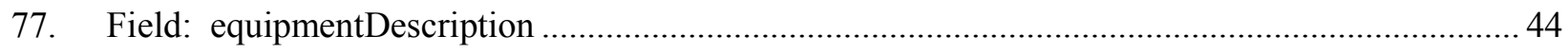




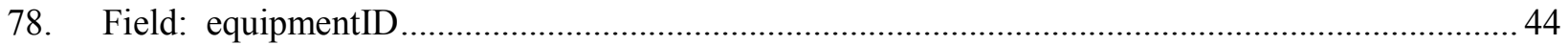

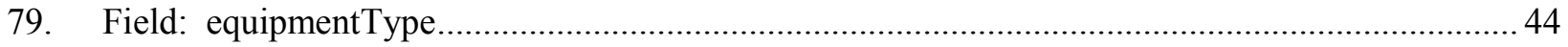

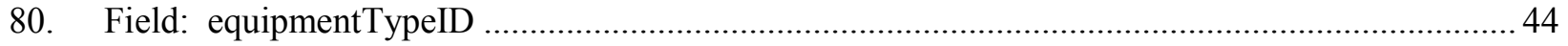

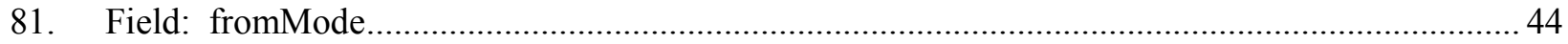

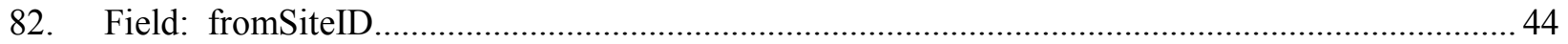

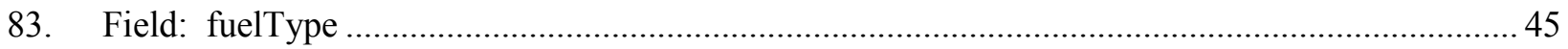

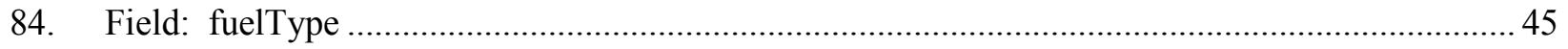

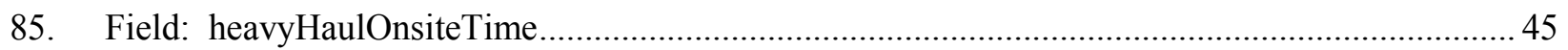

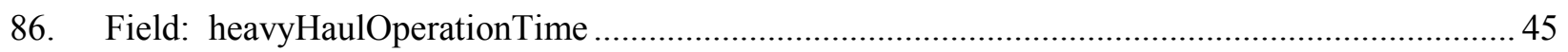

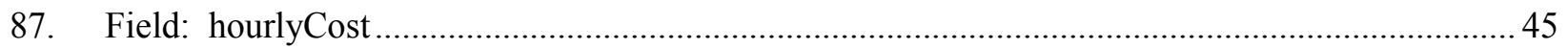

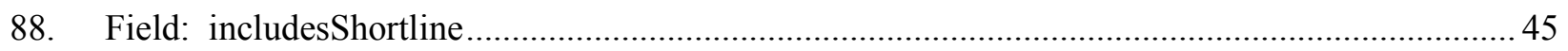

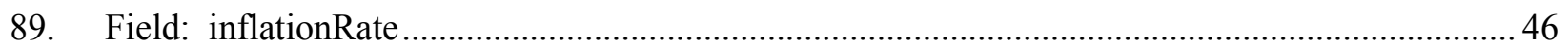

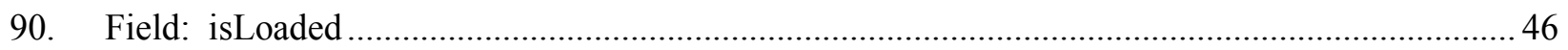

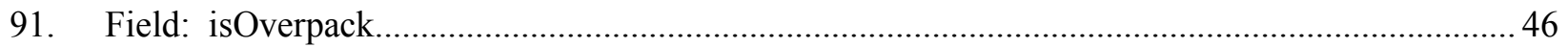

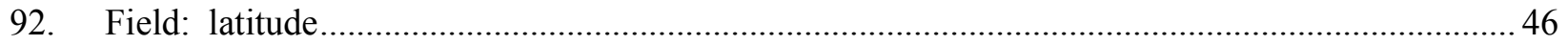

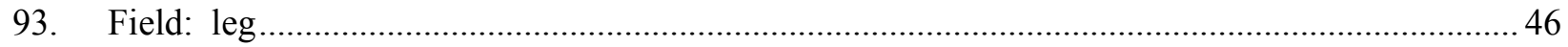

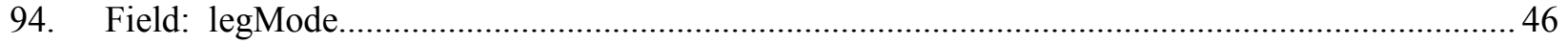

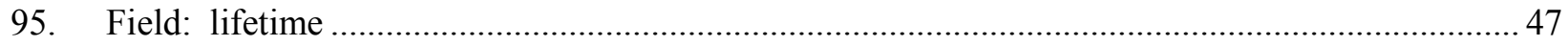

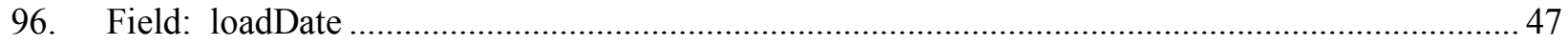

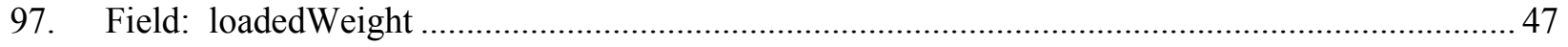

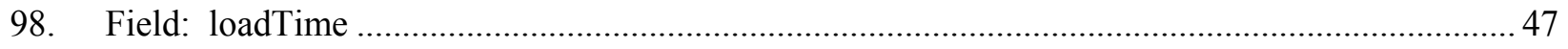

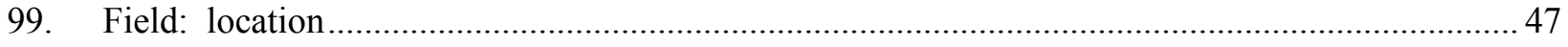

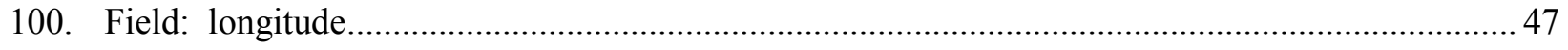

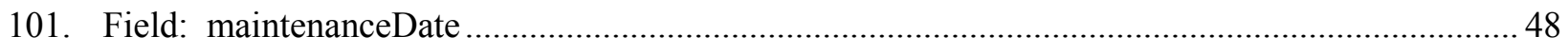

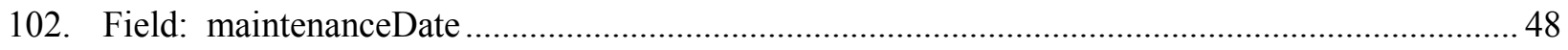

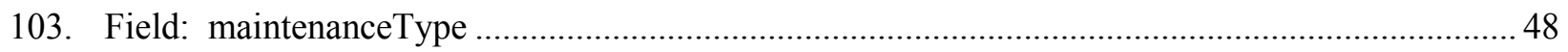




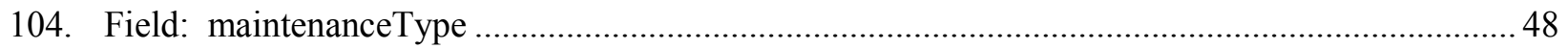

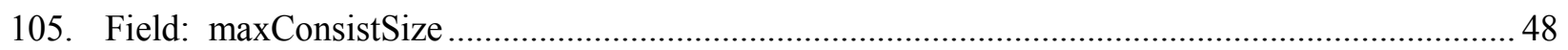

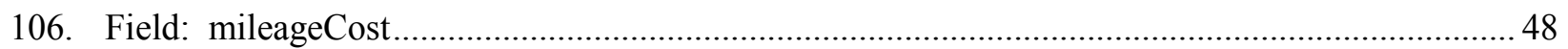

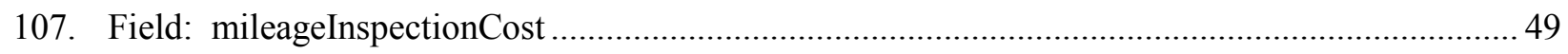

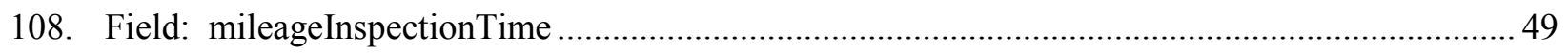

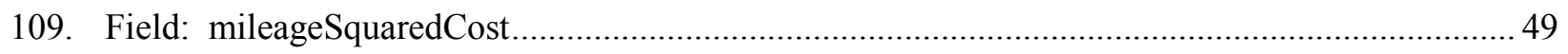

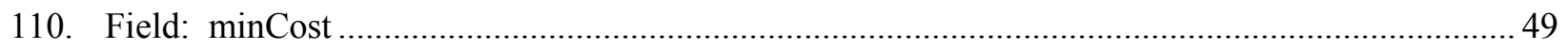

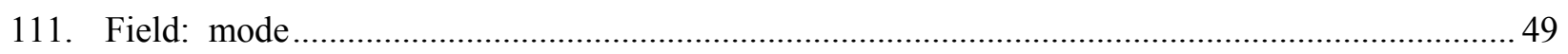

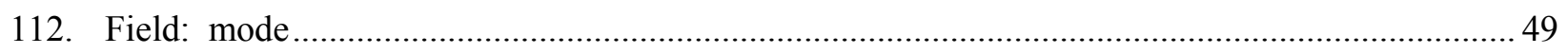

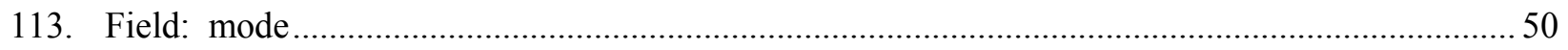

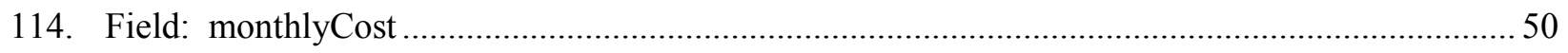

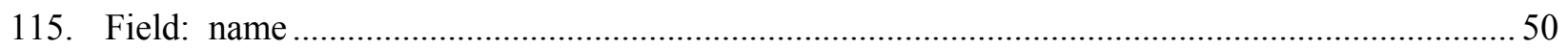

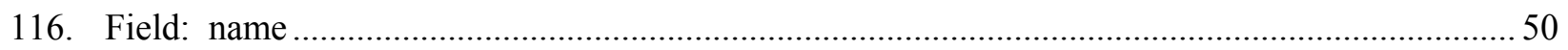

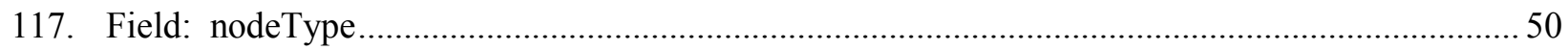

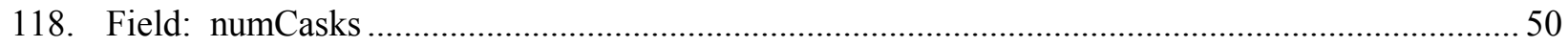

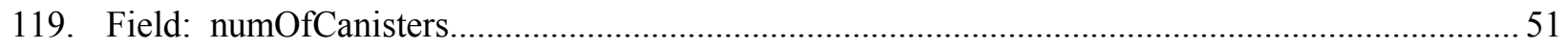

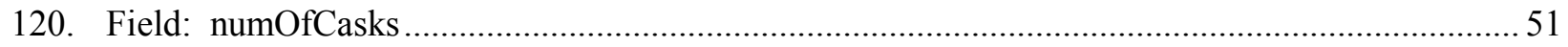

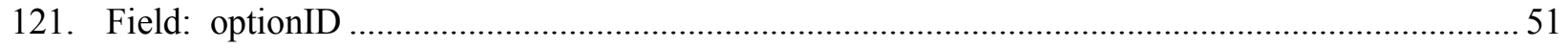

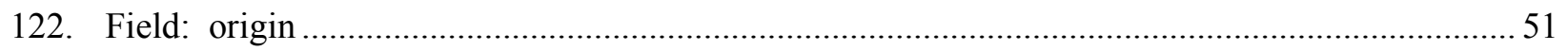

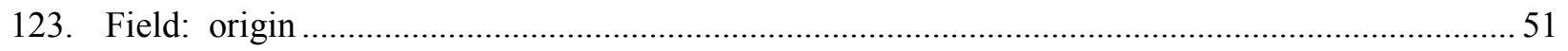

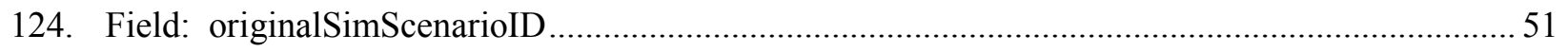

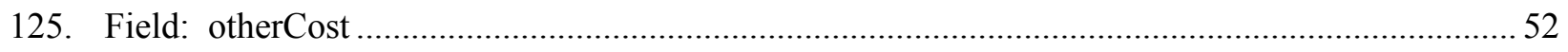

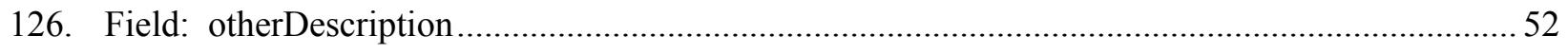

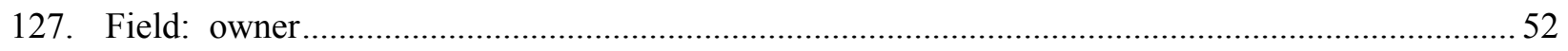

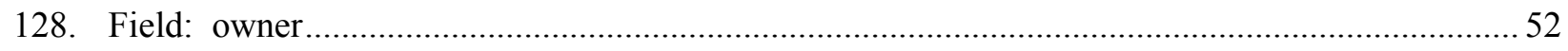

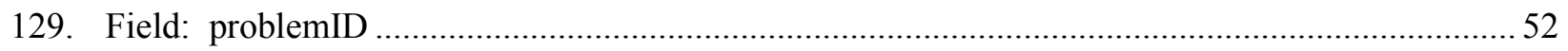




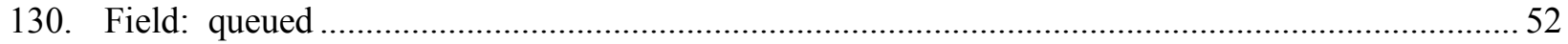

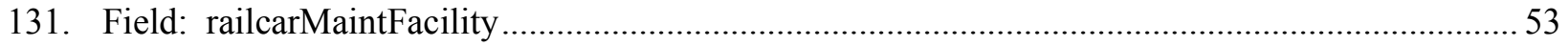

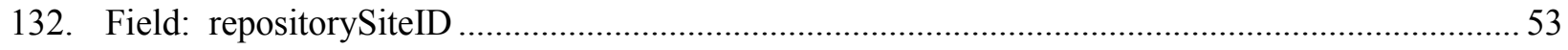

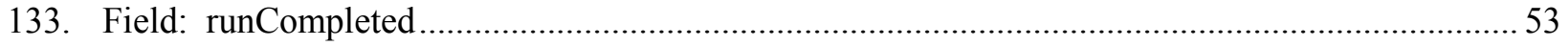

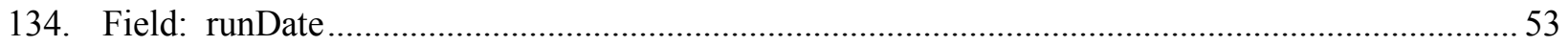

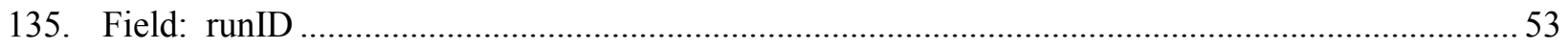

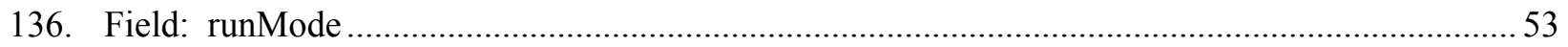

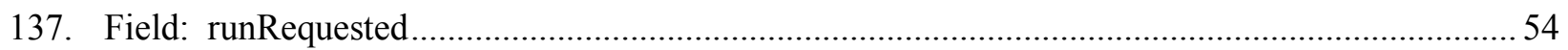

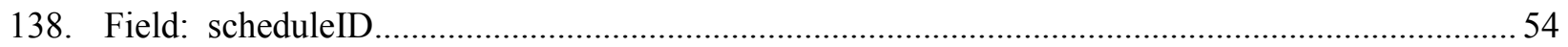

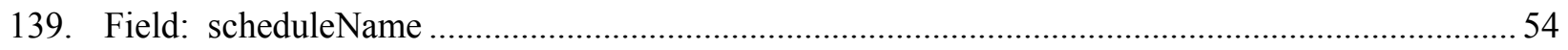

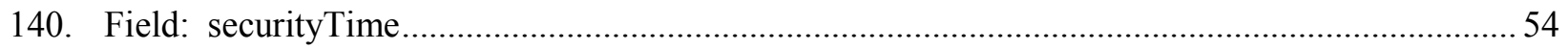

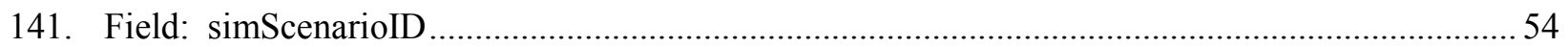

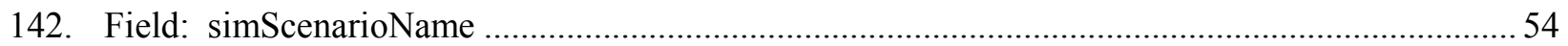

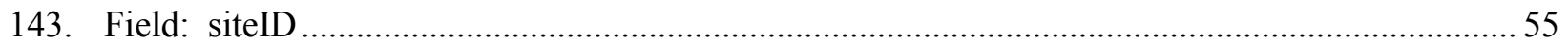

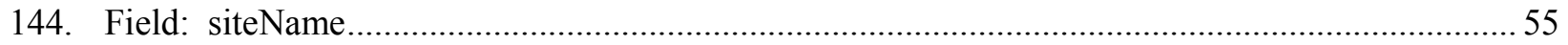

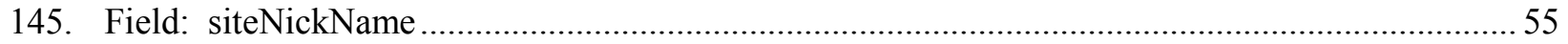

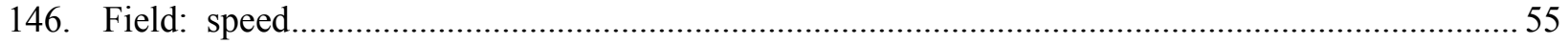

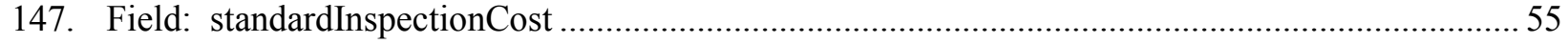

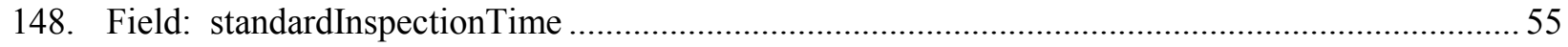

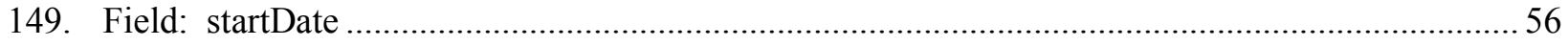

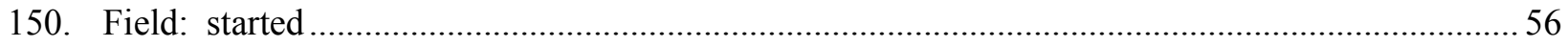

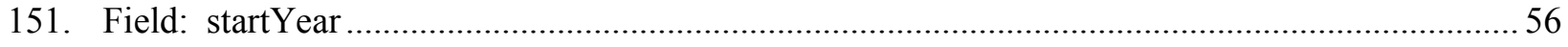

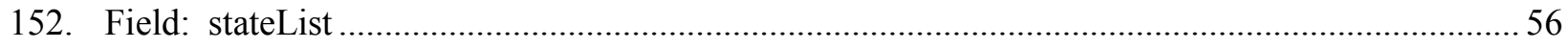

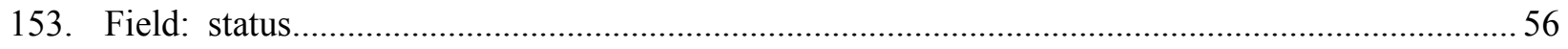

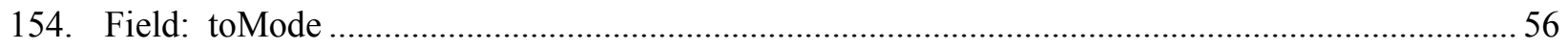

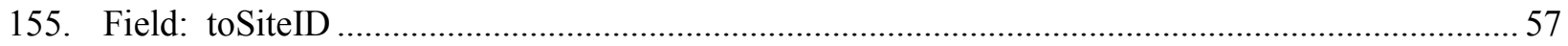




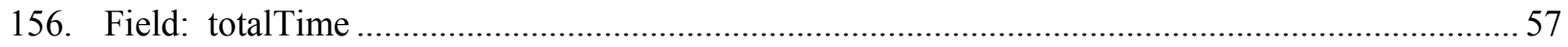

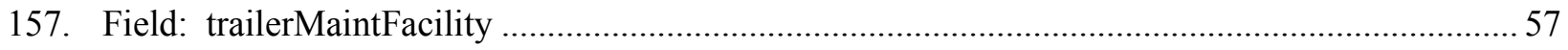

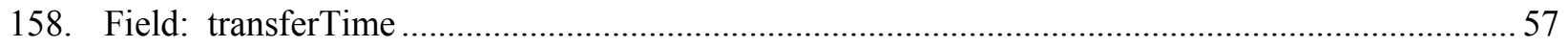

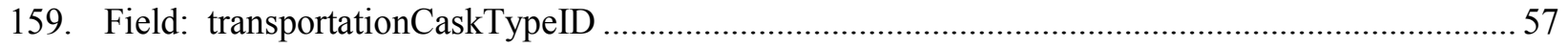

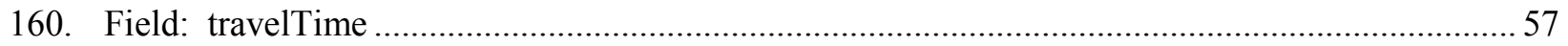

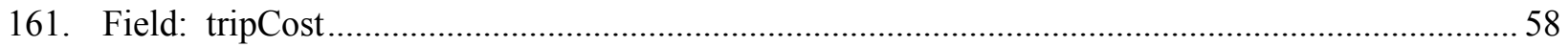

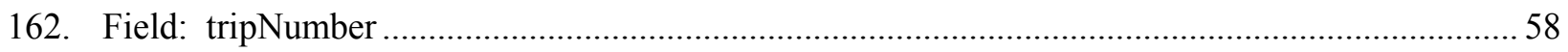

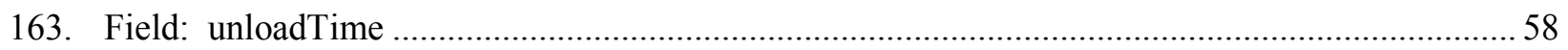

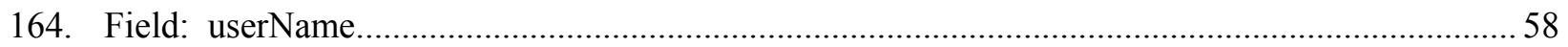

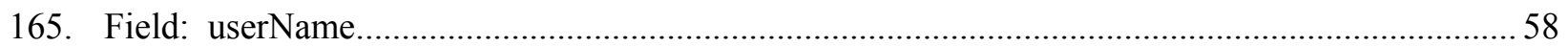

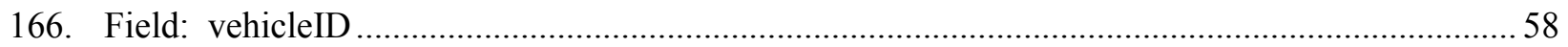

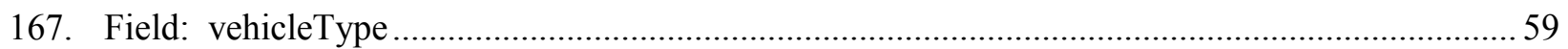

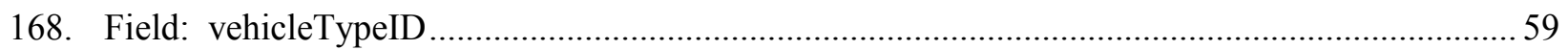

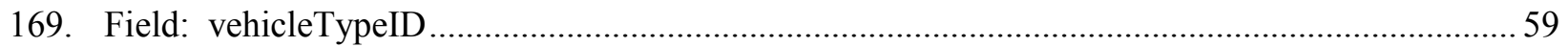

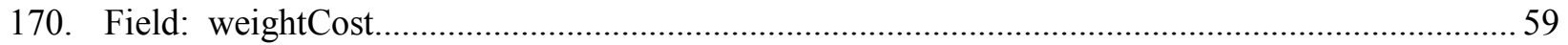

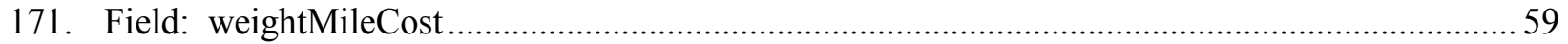

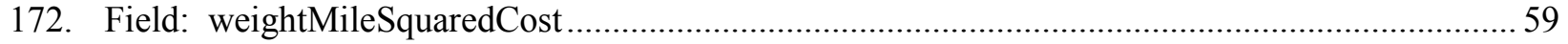

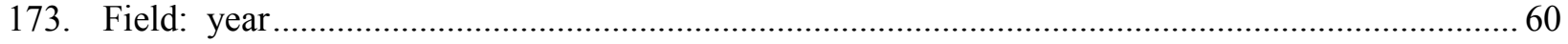

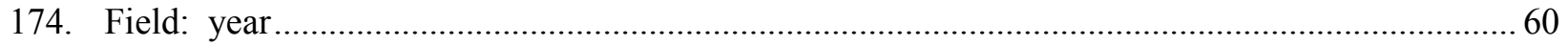


Transportation Storage Logistics (TSL) Model Data Management Manual October 2012 


\section{TRANSPORTATION STORAGE LOGISTICS MODEL DATA MANAGEMENT MANUAL}

\section{Canister \\ Definition:}

Information about canisters. In TOM, a canister is something that stores assemblies, but needs an overpack to be transported.

\begin{tabular}{|c|c|c|c|}
\hline $\begin{array}{c}\text { Primary } \\
\text { Key }\end{array}$ & Column Name & Type & Description \\
\hline \multirow[t]{7}{*}{$\mathbf{x}$} & canisterTypeID & int & $\begin{array}{l}\text { canister type ID } \\
\text { number }\end{array}$ \\
\hline & canisterName & nvarchar & canister name \\
\hline & transportationCaskTypeID & int & $\begin{array}{l}\text { cask type ID number } \\
\text { of transportation } \\
\text { overpack }\end{array}$ \\
\hline & assemblyCapacity & int & $\begin{array}{l}\text { number of } \\
\text { assemblies }\end{array}$ \\
\hline & fuelType & char & $\begin{array}{l}\text { fuel type (B = BWR, } P \\
=\text { PWR) }\end{array}$ \\
\hline & capitalCost & float & $\begin{array}{l}\text { acquisition cost to } \\
\text { purchase (\$) }\end{array}$ \\
\hline & calvinCaskID & int & $\begin{array}{l}\text { cask ID number in } \\
\text { CALVIN database }\end{array}$ \\
\hline
\end{tabular}




\section{Cask}

\section{Definition:}

Information about the casks. In TOM, a cask is a container that does not require a transportation overpack

\begin{tabular}{|c|c|c|c|}
\hline $\begin{array}{c}\text { Primary } \\
\text { Key }\end{array}$ & Column Name & Type & Description \\
\hline \multirow[t]{16}{*}{$x$} & caskTypeID & int & cask type ID number \\
\hline & caskName & nvarchar & cask name \\
\hline & description & nvarchar & cask description \\
\hline & mode & char & $\begin{array}{l}\text { usage mode }(\mathrm{R}=\text { rail } \\
\text { transportation, } \mathrm{T}= \\
\text { truck transportation, } \mathrm{S} \\
=\text { storage) }\end{array}$ \\
\hline & isOverpack & bit & $\begin{array}{l}\text { true if cask is an } \\
\text { overpack }\end{array}$ \\
\hline & fuelType & char & $\begin{array}{l}\text { fuel type }(B=B W R, P= \\
P W R, H=?, X=\text { ?) }\end{array}$ \\
\hline & assemblyCapacity & int & number of assemblies \\
\hline & emptyWeight & float & $\begin{array}{l}\text { empty cask weight (in } \\
\text { pounds) }\end{array}$ \\
\hline & loadedWeight & float & $\begin{array}{l}\text { fully loaded cask } \\
\text { weight (in pounds) }\end{array}$ \\
\hline & capitalCost & float & $\begin{array}{l}\text { acquisition cost to } \\
\text { purchase (\$) }\end{array}$ \\
\hline & standardInspectionTime & smallint & $\begin{array}{l}\text { inspection time after } \\
\text { each trip (hours) }\end{array}$ \\
\hline & standardlnspectionCost & float & $\begin{array}{l}\text { inspection cost after } \\
\text { each trip (\$) }\end{array}$ \\
\hline & annuallnspectionTime & smallint & $\begin{array}{l}\text { annual inspection time } \\
\text { (hours) }\end{array}$ \\
\hline & annuallnspectionCost & float & $\begin{array}{l}\text { annual inspection cost } \\
\text { (\$) }\end{array}$ \\
\hline & calvinCaskID & int & $\begin{array}{l}\text { cask ID number in } \\
\text { CALVIN database }\end{array}$ \\
\hline & loadTime & int & $\begin{array}{l}\text { time to load cask } \\
\text { (hours) }\end{array}$ \\
\hline
\end{tabular}


Transportation Storage Logistics (TSL) Model Data Management Manual October 2012

\begin{tabular}{|lll|}
\hline unloadTime & int & $\begin{array}{l}\text { time to unload cask } \\
\text { (hours) }\end{array}$ \\
\hline lifetime & int & $\begin{array}{l}\text { usable life of cask } \\
\text { (years) }\end{array}$ \\
\hline decommissioningCost & float & $\begin{array}{l}\text { cost to dispose of cask } \\
\text { (\$) }\end{array}$ \\
\hline
\end{tabular}




\section{Cost}

\section{Definition:}

Information about the costs in the system. Fields exist for specifying the costs as a function of different attributes of transportation. Any or all of these can be used.

\begin{tabular}{|c|c|c|c|}
\hline $\begin{array}{c}\text { Primary } \\
\text { Key }\end{array}$ & Column Name & Type & Description \\
\hline$x$ & mode & nvarchar & $\begin{array}{l}\text { transportation mode } \\
\text { ( } H=\text { highway, } L=\text { local, } \\
R=\text { rail) }\end{array}$ \\
\hline \multirow[t]{15}{*}{$x$} & detail & nvarchar & cost description \\
\hline & year & int & $\begin{array}{l}\text { calendar year of cost } \\
\text { (used in inflation } \\
\text { calculations) }\end{array}$ \\
\hline & tripCost & float & cost per trip \\
\hline & carCost & float & cost per car \\
\hline & mileageCost & float & cost per mile \\
\hline & mileageSquaredCost & float & cost per mile squared \\
\hline & monthlyCost & float & cost per month \\
\hline & dailyCost & float & cost per day \\
\hline & hourlyCost & float & cost per hour \\
\hline & weightCost & float & cost per ton \\
\hline & weightMileCost & float & cost per ton mile \\
\hline & weightMileSquaredCost & float & $\begin{array}{l}\text { cost per ton mile } \\
\text { squared }\end{array}$ \\
\hline & minCost & float & minimum cost \\
\hline & otherCost & float & other cost \\
\hline & otherDescription & nvarchar & other cost description \\
\hline
\end{tabular}




\section{Equipment} Definition:

Information about the equipment available to use.

\begin{tabular}{|clll|}
\hline $\begin{array}{c}\text { Primary } \\
\text { Key }\end{array}$ & Column Name & Type & Description \\
\hline $\mathbf{X}$ & equipmentDescription & nvarchar & description \\
\hline & speed & smallint & $\begin{array}{l}\text { speed of equipment } \\
\text { (mph) }\end{array}$ \\
\hline
\end{tabular}




\section{Inflation}

\section{Definition:}

Information about the yearly inflation rate.

\begin{tabular}{|clll|}
\hline $\begin{array}{c}\text { Primary } \\
\text { Key }\end{array}$ & $\begin{array}{l}\text { Column } \\
\text { Name }\end{array}$ & Type & Description \\
\hline $\mathbf{X}$ & year & int & calendar year \\
\hline inflationRate & float & $\begin{array}{l}\text { yearly inflation rate (as a percent, so } \\
\text { e.g., } 5.2 \%=5.2 \text { ) }\end{array}$ \\
\hline
\end{tabular}


6. Job

\section{Definition:}

The job queue for TOM runs. The users place jobs into the queue, and an automatic process picks up the job and runs it.

\begin{tabular}{|clll|}
\hline $\begin{array}{c}\text { Primary } \\
\text { Key }\end{array}$ & $\begin{array}{l}\text { Column } \\
\text { Name }\end{array}$ & Type & Description \\
\hline $\mathbf{X}$ & runID & int & run ID number \\
\hline $\mathbf{X}$ & userName & nvarchar & user who added job to queue \\
\hline & queued & datetime & $\begin{array}{l}\text { date job was added to the } \\
\text { queue }\end{array}$ \\
\hline started & datetime & date job was started \\
\hline completed & datetime & date job was completed \\
\hline status & nvarchar & $\begin{array}{l}\text { job status (queued, started, } \\
\text { finished) }\end{array}$ \\
\hline
\end{tabular}




\section{PickupSchedule Definition:}

The pickup schedules generated by CALVIN.

\begin{tabular}{|llll|}
\hline $\begin{array}{c}\text { Primary } \\
\text { Key }\end{array}$ & Column Name & Type & Description \\
\hline $\mathbf{X}$ & scheduleID & int & schedule ID number \\
\hline scheduleName & nvarchar & schedule name \\
\hline owner & nvarchar & $\begin{array}{l}\text { owner (user who input } \\
\text { schedule) }\end{array}$ \\
\hline loadDate & datetime & $\begin{array}{l}\text { date that schedule was } \\
\text { loaded }\end{array}$ \\
\hline
\end{tabular}




\section{PickupScheduleCanister}

Definition:

Canister pickup demands that were generated by CALVIN.

\begin{tabular}{|clll|}
\hline $\begin{array}{c}\text { Primary } \\
\text { Key }\end{array}$ & Column Name & Description \\
\hline $\mathbf{X}$ & scheduleID & int & schedule ID number \\
\hline $\mathbf{X}$ & origin & nvarchar & $\begin{array}{l}\text { pickup location (either } \\
\text { reactor site ID number, or } \\
\text { ISF) }\end{array}$ \\
\hline $\mathbf{X}$ & year & int & calendar year \\
\hline $\mathbf{X}$ & destination & nvarchar & $\begin{array}{l}\text { destination location (either } \\
\text { ISF or MGR) }\end{array}$ \\
\hline $\mathbf{X}$ & canisterTypelD & int & canister type ID number \\
\hline $\mathbf{X}$ & alreadylnCanister & bit & true of fuel is in a canister \\
\hline $\mathbf{X}$ & location & location of fuel (P $=$ pool, $D=$ \\
& dry storage)
\end{tabular}




\section{PickupScheduleCask}

\section{Definition:}

Cask pickup demands generated by CALVIN.

\begin{tabular}{|clll|}
\hline $\begin{array}{c}\text { Primary } \\
\text { Key }\end{array}$ & Column Name & Type & Description \\
\hline $\mathbf{X}$ & scheduleID & int & schedule ID number \\
\hline $\mathbf{X}$ & origin & nvarchar & $\begin{array}{l}\text { pickup location (either } \\
\text { reactor site ID number, or } \\
\text { ISF) }\end{array}$ \\
\hline $\mathbf{X}$ & year & int & calendar year \\
\hline $\mathbf{X}$ & destination & nvarchar & $\begin{array}{l}\text { destination location (either } \\
\text { ISF or MGR) }\end{array}$ \\
\hline $\mathbf{X}$ & caskTypelD & int & cask type ID number \\
\hline $\mathbf{X}$ & alreadylnCanister & bit & true of fuel is in a canister \\
\hline $\mathbf{X}$ & alreadylnCask & bit & true if fuel is in a cask \\
\hline $\mathbf{X}$ & location & nvarchar & $\begin{array}{l}\text { location of fuel }(\mathrm{P}=\text { pool, } \mathrm{D}= \\
\text { dry storage) }\end{array}$ \\
\hline & numOfCasks & int & $\begin{array}{l}\text { number of casks to be picked } \\
\text { up }\end{array}$ \\
\hline & & &
\end{tabular}




\section{PickupSchedulePermission}

\section{Definition:}

Permission set used to restrict users from accessing, modifying, or deleting other users' data.

\begin{tabular}{|c|c|c|c|}
\hline $\begin{array}{c}\text { Primary } \\
\text { Key }\end{array}$ & $\begin{array}{l}\text { Column } \\
\text { Name }\end{array}$ & Type & Description \\
\hline$x$ & scheduleID & int & schedule ID number \\
\hline $\mathbf{x}$ & userName & nvarchar & user name \\
\hline & canRead & bit & $\begin{array}{l}\text { true if user has read } \\
\text { permission }\end{array}$ \\
\hline & canWrite & bit & $\begin{array}{l}\text { true if user has write } \\
\text { permission }\end{array}$ \\
\hline
\end{tabular}




\section{Scenario}

\section{Definition:}

Information about a TOM run. Contains non-site-specific information, as well as default site information.

\begin{tabular}{|c|c|c|c|}
\hline $\begin{array}{l}\text { Primary } \\
\text { Key }\end{array}$ & Column Name & Type & Description \\
\hline $\mathbf{x}$ & runID & int & run ID number \\
\hline & name & nvarchar & run name \\
\hline & owner & nvarchar & $\begin{array}{l}\text { owner (user who created } \\
\text { the run) }\end{array}$ \\
\hline & startYear & int & analysis start year \\
\hline & endYear & int & analysis end year \\
\hline & scheduleID & int & schedule ID number \\
\hline & caskMaintFacility & int & $\begin{array}{l}\text { site ID of cask maintenance } \\
\text { facility }\end{array}$ \\
\hline & railcarMaintFacility & int & $\begin{array}{l}\text { site ID of railcar } \\
\text { maintenance facility }\end{array}$ \\
\hline & trailerMaintFacility & int & $\begin{array}{l}\text { site ID of truck trailer } \\
\text { maintenance facility }\end{array}$ \\
\hline & repositorySiteID & int & site ID of repository \\
\hline & defaultConsistSize & int & $\begin{array}{l}\text { default consist size (can be } \\
\text { overridden by consistSize } \\
\text { field in the ScenarioSite } \\
\text { table) }\end{array}$ \\
\hline & costYear & int & $\begin{array}{l}\text { base year for cost inflation } \\
\text { (set to current year to } \\
\text { inflate past years' costs to } \\
\text { current dollars) }\end{array}$ \\
\hline & runRequested & bit & $\begin{array}{l}\text { true if a run request has } \\
\text { been made }\end{array}$ \\
\hline & runCompleted & bit & $\begin{array}{l}\text { true if the run has } \\
\text { completed }\end{array}$ \\
\hline & runDate & datetime & date of the last run \\
\hline & runMode & nvarchar & $\begin{array}{l}\text { run mode for scheduling } \\
\text { program }(Q=\text { quick, } O= \\
\text { optimized })\end{array}$ \\
\hline
\end{tabular}


Transportation Storage Logistics (TSL) Model Data Management Manual October 2012 


\section{ScenarioAcquisition}

Definition:

Contains run results on the acquisition of assets, both cask and vehicles.

\begin{tabular}{|c|c|c|c|}
\hline $\begin{array}{l}\text { Primary } \\
\text { Key }\end{array}$ & Column Name & Type & Description \\
\hline $\mathbf{x}$ & runID & int & run ID number \\
\hline$x$ & acquisitionDate & datetime & date acquired \\
\hline $\mathbf{x}$ & equipmentType & nvarchar & $\begin{array}{l}\text { category of equipment (CASK } \\
\text { or VEHICLE) }\end{array}$ \\
\hline$x$ & equipmentTypeID & int & $\begin{array}{l}\text { equipment type ID number } \\
\text { (cask ID number for CASK } \\
\text { assets, and vehicle ID } \\
\text { number of VEHICLE assets) }\end{array}$ \\
\hline$x$ & equipmentID & int & $\begin{array}{l}\text { equipment ID number } \\
\text { (sequential number for the } \\
\text { individual assets used for } \\
\text { tracking) }\end{array}$ \\
\hline
\end{tabular}




\section{ScenarioCaskMaintenance}

\section{Definition:}

Contains run results on the cask maintenance performed.

\begin{tabular}{|clll|}
\hline $\begin{array}{c}\text { Primary } \\
\text { Key }\end{array}$ & Column Name & Type & Description \\
\hline $\mathbf{X}$ & runID & int & run ID number \\
\hline $\mathbf{X}$ & caskTypeID & int & cask type ID number \\
\hline $\mathbf{X}$ & caskID & int & $\begin{array}{l}\text { cask ID number (internal ID } \\
\text { number assigned to an } \\
\text { individual cask to facilitate } \\
\text { tracking through shipments) }\end{array}$ \\
\hline $\mathbf{X}$ & maintenanceDate & datetime & date of maintenance \\
\hline & maintenanceType & nvarchar & $\begin{array}{l}\text { type of maintenance } \\
\text { (standard or annual) }\end{array}$ \\
\hline
\end{tabular}




\section{ScenarioCost}

\section{Definition:}

Contains run results on the yearly costs incurred in the transportation process.

\begin{tabular}{|clll|}
\hline $\begin{array}{c}\text { Primary } \\
\text { Key }\end{array}$ & $\begin{array}{l}\text { Column } \\
\text { Name }\end{array}$ & Type & Description \\
\hline $\mathbf{X}$ & runID & int & run ID number \\
\hline $\mathbf{X}$ & calendarYear & int & calendar year \\
\hline $\mathbf{X}$ & costCategory & nvarchar & $\begin{array}{l}\text { cost category (Capital, } \\
\text { Maintenance, or Operations) }\end{array}$ \\
\hline $\mathbf{X}$ & costDetail & nvarchar & cost detail \\
\hline & cost & float & cost $(\$)$ \\
\hline
\end{tabular}




\section{ScenarioErrorLog}

\section{Definition:}

Contains any error messages generated during the TOM run.

\begin{tabular}{|clll|}
\hline $\begin{array}{c}\text { Primary } \\
\text { Key }\end{array}$ & $\begin{array}{l}\text { Column } \\
\text { Name }\end{array}$ & Type & Description \\
\hline $\mathbf{X}$ & runID & int & run ID number \\
\hline $\mathbf{X}$ & problemID & int & $\begin{array}{l}\text { problem ID number (sequential } \\
\text { ID for run) }\end{array}$ \\
\hline & description & nvarchar & problem description \\
\hline
\end{tabular}


16. ScenarioISF

\section{Definition:}

Contains the site IDs of the ISFs designated for a run. Currently the interface allows for the specification of one ISF per run, but the TOM model allows multiple ISFs.

\begin{tabular}{|clll|}
\hline Primary Key & Column Name & Type & Description \\
\hline $\mathbf{X}$ & runID & int & run ID number \\
\hline $\mathbf{X}$ & sitelD & int & site ID number \\
\hline
\end{tabular}




\section{ScenarioPermission}

\section{Definition:}

Permission set used to restrict users from accessing, modifying, or deleting other users' data.

\begin{tabular}{|clll|}
\hline $\begin{array}{c}\text { Primary } \\
\text { Key }\end{array}$ & $\begin{array}{l}\text { Column } \\
\text { Name }\end{array}$ & Type & Description \\
\hline $\mathbf{X}$ & runID & int & run ID number \\
\hline $\mathbf{X}$ & userName & nvarchar & user name \\
\hline & canRead & bit & $\begin{array}{l}\text { true if user has read } \\
\text { permission }\end{array}$ \\
\hline canWrite & bit & $\begin{array}{l}\text { true if user has write } \\
\text { permission }\end{array}$ \\
\hline
\end{tabular}




\section{ScenarioSite}

\section{Definition:}

Site-specific information for a run. Allows user to override the default constist size specified in the Scenario table.

\begin{tabular}{|clll|}
\hline $\begin{array}{c}\text { Primary } \\
\text { Key }\end{array}$ & Column Name & Type & Description \\
\hline $\mathbf{X}$ & runID & int & run ID number \\
\hline $\mathbf{X}$ & sitelD & int & site ID number \\
\hline maxConsistSize & int & $\begin{array}{l}\text { maximum consist size (overwrites } \\
\text { defaultConsistSize from the } \\
\text { Scenario table) }\end{array}$ \\
\hline
\end{tabular}




\section{ScenarioVehicleMaintenance}

\section{Definition:}

Run results on the vehicle maintenance performed during the transportation process.

\begin{tabular}{|c|c|c|c|}
\hline $\begin{array}{l}\text { Primary } \\
\text { Key }\end{array}$ & Column Name & Type & Description \\
\hline$x$ & runID & int & run ID number \\
\hline $\mathbf{x}$ & vehicleTypeID & int & vehicle type ID number \\
\hline $\mathbf{x}$ & vehicleID & int & $\begin{array}{l}\text { vehicle ID number (internal } \\
\text { ID number assigned to an } \\
\text { individual vehicle to facilitate } \\
\text { tracking through shipments) }\end{array}$ \\
\hline \multirow[t]{2}{*}{$\mathbf{x}$} & maintenanceDate & datetime & maintenance date \\
\hline & maintenanceType & nvarchar & $\begin{array}{l}\text { type of maintenance } \\
\text { (standard or mileage) }\end{array}$ \\
\hline
\end{tabular}




\section{SimScenario}

\section{Definition:}

Information on the simulation scenarios. Simulations were run to develop times for at-reactor operations involving ancillary equipment, such as cranes and heavy haul trucks.

\begin{tabular}{|c|c|c|c|}
\hline $\begin{array}{l}\text { Primary } \\
\text { Key }\end{array}$ & Column Name & Type & Description \\
\hline$x$ & simScenariolD & smallint & $\begin{array}{l}\text { simulation scenario ID } \\
\text { number }\end{array}$ \\
\hline & simScenarioName & nvarchar & simulation scenario name \\
\hline
\end{tabular}




\section{SimulationOutput}

\section{Definition:}

Simulation results that describe the times needed for at-reactor operations for ancillary equipment, based on transportation scenario and size of consist.

\begin{tabular}{|c|c|c|c|}
\hline $\begin{array}{l}\text { Primary } \\
\text { Key }\end{array}$ & Column Name & Type & Description \\
\hline $\mathbf{x}$ & simScenariolD & smallint & $\begin{array}{l}\text { simulation scenario ID } \\
\text { number }\end{array}$ \\
\hline \multirow[t]{10}{*}{$x$} & numCasks & smallint & consist size \\
\hline & craneOperationTime & float & $\begin{array}{l}\text { time crane is in use } \\
\text { (hours) }\end{array}$ \\
\hline & craneOnsiteTime & float & $\begin{array}{l}\text { time crane is onsite } \\
\text { (hours) }\end{array}$ \\
\hline & heavyHaulOperationTime & float & $\begin{array}{l}\text { time } \mathrm{HH} \text { is in operation } \\
\text { (hours) }\end{array}$ \\
\hline & heavyHaulOnsiteTime & float & $\begin{array}{l}\text { time } \mathrm{HH} \text { is onsite } \\
\text { (hours) }\end{array}$ \\
\hline & bargeLoadingTime & float & $\begin{array}{l}\text { time to load barges } \\
\text { (hours) }\end{array}$ \\
\hline & bargeUnloadingTIme & float & $\begin{array}{l}\text { time to unload barges } \\
\text { (hours) }\end{array}$ \\
\hline & bargeWaitTime & float & $\begin{array}{l}\text { time barges are } \\
\text { waiting (hours) }\end{array}$ \\
\hline & securityTime & float & $\begin{array}{l}\text { time before departure } \\
\text { that security must be } \\
\text { present (hours) }\end{array}$ \\
\hline & totalTime & float & $\begin{array}{l}\text { total time for loading } \\
\text { operation (minus } \\
\text { loading fuel into casks } \\
\text { and/or canisters) } \\
\text { (hours) }\end{array}$ \\
\hline
\end{tabular}




\section{SimulationTransferTime}

\section{Definition:}

Broad level information on times needed to transfer a cask from one mode to another. This table is included for reference purposes. It is not being accessed by TSL.

\begin{tabular}{|clll|}
\hline $\begin{array}{c}\text { Primary } \\
\text { Key }\end{array}$ & $\begin{array}{l}\text { Column } \\
\text { Name }\end{array}$ & Type & Description \\
\hline $\mathbf{X}$ & fromMode & nvarchar & $\begin{array}{l}\text { mode from which the cask is } \\
\text { transferred }\end{array}$ \\
\hline $\mathbf{X}$ & toMode & nvarchar & $\begin{array}{l}\text { mode to which the cask is } \\
\text { transferred }\end{array}$ \\
\hline $\mathbf{X}$ & isLoaded & bit & true if the cask is loaded \\
\hline & transferTime & float & transfer time (hours) \\
\hline
\end{tabular}


23. Site

\section{Definition:}

Site information that is common to all scenarios. Users may add sites to the system, but are not allowed to modify existing site information.

\begin{tabular}{|llll|}
\hline $\begin{array}{c}\text { Primary } \\
\text { Key }\end{array}$ & Column Name & Type & Description \\
\hline $\mathbf{X}$ & sitelD & int & site ID number \\
\hline simScenarioID & smallint & $\begin{array}{l}\text { simulation scenario ID } \\
\text { number }\end{array}$ \\
\hline siteName & nvarchar & site name \\
\hline latitude & float & latitude (degrees) \\
\hline longitude & float & longitude (degrees) \\
\hline originalSimScenariolD & int & < unused> \\
\hline
\end{tabular}




\section{SiteDistance}

\section{Definition:}

Site-to-site distance and travel time information, broken down by mode. Note that the rail mode may include water travel (by barge) and highway travel (by heavy haul).

\begin{tabular}{|c|c|c|c|}
\hline $\begin{array}{l}\text { Primary } \\
\text { Key }\end{array}$ & Column Name & Type & Description \\
\hline $\mathbf{x}$ & fromSitelD & int & site ID number \\
\hline $\mathbf{x}$ & toSiteID & int & site ID number \\
\hline $\mathbf{x}$ & optionID & int & routing option ID number \\
\hline $\mathbf{x}$ & mode & nvarchar & $\begin{array}{l}\text { overall mode of travel }(\mathrm{H}= \\
\text { highway, } \mathrm{R}=\text { rail) }\end{array}$ \\
\hline \multirow[t]{7}{*}{$x$} & leg & int & leg number \\
\hline & distance & float & leg distance (miles) \\
\hline & travelTime & float & leg travel time (hours) \\
\hline & legMode & nvarchar & $\begin{array}{l}\text { mode for leg }(H=\text { highway, } \mathrm{R} \\
=\text { rail, } \mathrm{W}=\text { waterway) }\end{array}$ \\
\hline & statelist & nvarchar & $\begin{array}{l}\text { FIPS codes of states } \\
\text { traversed }\end{array}$ \\
\hline & derivation & nvarchar & $\begin{array}{l}\text { source of data }(\mathrm{C}=\mathrm{CTA} \\
\text { network, } \mathrm{E}=\text { estimate, } \mathrm{T}= \\
\text { TRAGIS) }\end{array}$ \\
\hline & includesShortline & bit & $\begin{array}{l}\text { true if shortline rail is } \\
\text { included in route }\end{array}$ \\
\hline
\end{tabular}




\section{SiteNickname}

\section{Definition:}

Nicknames for sites. Is provided to eliminate duplicate sites in the database, and is generally used for alternate spellings or abbreviations of site names. Entries are added during the schedule load process.

\begin{tabular}{|clll|}
\hline Primary Key & Column Name & Type & Description \\
\hline $\mathbf{X}$ & siteNickName & nvarchar & site nickname \\
\hline sitelD & int & site ID number \\
\hline
\end{tabular}




\section{SiteNode}

\section{Definition:}

The transportation locations associate with each site. These may include road access, rail access, waterway access, and mode transfer locations.

\begin{tabular}{|clll|}
\hline $\begin{array}{c}\text { Primary } \\
\text { Key }\end{array}$ & $\begin{array}{l}\text { Column } \\
\text { Name }\end{array}$ & Type & Description \\
\hline $\mathbf{X}$ & sitelD & int & site ID number \\
\hline $\mathbf{X}$ & optionID & int & routing option ID number \\
\hline $\mathbf{X}$ & nodeType & nvarchar & $\begin{array}{l}\text { node type (road, water dock, rail, } \\
\text { water rail, rail siding) }\end{array}$ \\
\hline & latitude & float & latitude (degrees) \\
\hline & longitude & float & longitude (degrees) \\
\hline
\end{tabular}




\section{SiteTransportationOption}

\section{Definition:}

Transportation options for sites. Currently all sites have only one mode for access. This table (and in general, the transportation optionID field) is included to allow the analyst to investigate how the transportation plan and requirements change as site access modes improve or deteriorate.

\begin{tabular}{|clll|}
\hline Primary Key & Column Name & Type & Description \\
\hline $\mathbf{X}$ & sitelD & int & site ID number \\
\hline $\mathbf{X}$ & optionID & int & routing option ID number \\
\hline & name & nvarchar & routing option name \\
\hline
\end{tabular}




\section{Trip}

\section{Definition:}

Contains run results on the trips generated by TOM.

\begin{tabular}{|clll|}
\hline $\begin{array}{c}\text { Primary } \\
\text { Key }\end{array}$ & $\begin{array}{l}\text { Column } \\
\text { Name }\end{array}$ & Type & Description \\
\hline $\mathbf{X}$ & runID & int & run ID number \\
\hline $\mathbf{X}$ & tripNumber & int & trip number (internal) \\
\hline origin & nvarchar & $\begin{array}{l}\text { origin location (either reactor } \\
\text { site ID number or ISF) }\end{array}$ \\
\hline & destination & nvarchar & $\begin{array}{l}\text { destination location (either ISF } \\
\text { or MGR) }\end{array}$ \\
\hline
\end{tabular}




\section{TripCaskXref}

\section{Definition:}

Contains run results on the casks involved in each trip.

\begin{tabular}{|clll|}
\hline $\begin{array}{c}\text { Primary } \\
\text { Key }\end{array}$ & $\begin{array}{l}\text { Column } \\
\text { Name }\end{array}$ & Type & Description \\
\hline $\mathbf{X}$ & runID & int & run ID number \\
\hline $\mathbf{X}$ & tripNumber & int & trip number (internal) \\
\hline $\mathbf{X}$ & caskTypeID & int & cask type ID number \\
\hline $\mathbf{X}$ & caskID & int & $\begin{array}{l}\text { cask ID number (internal ID number } \\
\text { assigned to an individual cask to } \\
\text { facilitate tracking through shipments) }\end{array}$ \\
\hline
\end{tabular}




\section{TripCost}

\section{Definition:}

Contains run results on the cost of each trip.

\begin{tabular}{|clll|}
\hline $\begin{array}{c}\text { Primary } \\
\text { Key }\end{array}$ & $\begin{array}{l}\text { Column } \\
\text { Name }\end{array}$ & Type & Description \\
\hline $\mathbf{X}$ & runID & int & run ID number \\
\hline $\mathbf{X}$ & tripNumber & int & trip number (internal) \\
\hline $\mathbf{X}$ & costCategory & nvarchar & $\begin{array}{l}\text { cost category (Capital, } \\
\text { Maintenance, or Operations) }\end{array}$ \\
\hline $\mathbf{X}$ & costDetail & nvarchar & cost detail \\
\hline & cost & float & cost $(\$)$ \\
\hline
\end{tabular}




\section{TripTime}

\section{Definition:}

Contains run results on the timing of each activity during a trip.

\begin{tabular}{|c|c|c|c|}
\hline $\begin{array}{l}\text { Primary } \\
\text { Key }\end{array}$ & $\begin{array}{l}\text { Column } \\
\text { Name }\end{array}$ & Type & Description \\
\hline $\mathbf{x}$ & runID & int & run ID number \\
\hline$x$ & tripNumber & int & trip number (internal) \\
\hline$x$ & activity & nvarchar & $\begin{array}{l}\text { activity (CM = cask maintenance, } \\
\mathrm{FM}=\text { fleet maintenance, } \mathrm{LD}=\text { load, } \\
\mathrm{TC}=\text { travel to cask maintenance, } \mathrm{TF} \\
=\text { travel to fleet maintenance, } \mathrm{TR}= \\
\text { travel to reactor, } \mathrm{TS}=\text { travel to } \\
\text { storage, } \mathrm{UL}=\text { unload) }\end{array}$ \\
\hline & startDate & datetime & start date \\
\hline & endDate & datetime & end date \\
\hline
\end{tabular}




\section{TripVehicleXref}

\section{Definition:}

Contains run results on the vehicles used on each trip.

\begin{tabular}{|c|c|c|c|}
\hline $\begin{array}{c}\text { Primary } \\
\text { Key }\end{array}$ & $\begin{array}{l}\text { Column } \\
\text { Name }\end{array}$ & Type & Description \\
\hline$x$ & runID & int & run ID number \\
\hline $\mathbf{x}$ & tripNumber & int & trip number (internal) \\
\hline$x$ & vehicleTypeID & int & vehicle type ID number \\
\hline$x$ & vehicleID & int & $\begin{array}{l}\text { vehicle ID number (internal ID } \\
\text { number assigned to an individual } \\
\text { vehicle to facilitate tracking through } \\
\text { shipments) }\end{array}$ \\
\hline
\end{tabular}


Transportation Storage Logistics (TSL) Model Data Management Manual

October 2012

33. TSLUser

\section{Definition:}

The users of the TSL system.

\begin{tabular}{|clll|}
\hline Primary Key & Column Name & Type & Description \\
\hline $\mathbf{X}$ & userName & nvarchar & user name \\
\hline
\end{tabular}




\section{VehicleType}

\section{Definition:}

Information on the vehicles used in TOM.

\begin{tabular}{|c|c|c|c|}
\hline $\begin{array}{c}\text { Primary } \\
\text { Key }\end{array}$ & Column Name & Type & Description \\
\hline $\mathbf{x}$ & vehicleTypeID & int & $\begin{array}{l}\text { vehilce type ID } \\
\text { number }\end{array}$ \\
\hline & vehicleType & nvarchar & $\begin{array}{l}\text { vehicle type (R-Rail, T- } \\
\text { Truck) }\end{array}$ \\
\hline & description & nvarchar & vehicle description \\
\hline & capitalCost & float & $\begin{array}{l}\text { acquisition cost to } \\
\text { purchase (\$) }\end{array}$ \\
\hline & standardInspectionTime & smallint & $\begin{array}{l}\text { inspection time after } \\
\text { each trip (hours) }\end{array}$ \\
\hline & standardlnspectionCost & float & $\begin{array}{l}\text { inspection cost after } \\
\text { each trip (\$) }\end{array}$ \\
\hline & mileagelnspectionTime & smallint & $\begin{array}{l}\text { mileage inspection } \\
\text { time (hours) }\end{array}$ \\
\hline & mileagelnspectionCost & float & $\begin{array}{l}\text { mileage inspection } \\
\text { cost (\$) }\end{array}$ \\
\hline
\end{tabular}




\section{Field: acquisitionDate}

\section{Description:}

date acquired

\section{Tables:}

ScenarioAcquisition

36. Field: activity

\section{Description:}

activity $(\mathrm{CM}=$ cask maintenance, $\mathrm{FM}=$ fleet maintenance, $\mathrm{LD}=$ load, $\mathrm{TC}=$ travel to cask

maintenance, $\mathrm{TF}=$ travel to fleet maintenance, $\mathrm{TR}=$ travel to reactor, $\mathrm{TS}=$ travel to storage, $\mathrm{UL}$

$=$ unload)

\section{Tables:}

TripTime

\section{Field: alreadyInCanister}

\section{Description:}

true of fuel is in a canister

\section{Tables:}

PickupScheduleCanister, PickupScheduleCask

\section{Field: alreadylnCask}

\section{Description:}

true if fuel is in a cask

\section{Tables:}

PickupScheduleCask

\section{Field: annuallnspectionCost}

\section{Description:}

annual inspection cost (\$)

\section{Tables:}

Cask

\section{Field: annuallnspectionTime}

\section{Description:}

annual inspection time (hours)

\section{Tables:}

Cask 


\section{Field: assemblyCapacity}

\section{Description:}

number of assemblies

\section{Tables:}

Canister, Cask

\section{Field: bargeLoadingTime}

Description:

time to load barges (hours)

\section{Tables:}

SimulationOutput

43. Field: bargeUnloadingTIme

Description:

time to unload barges (hours)

\section{Tables:}

SimulationOutput

\section{Field: bargeWaitTime}

\section{Description:}

time barges are waiting (hours)

\section{Tables:}

SimulationOutput

\section{Field: calendarYear}

\section{Description:}

calendar year

\section{Tables:}

ScenarioCost

\section{Field: calvinCaskID}

\section{Description:}

cask ID number in CALVIN database

\section{Tables:}

Canister, Cask 
47. Field: canisterName

Description:

canister name

\section{Tables:}

Canister

\section{Field: canisterTypelD} Description:

canister type ID number

\section{Tables:}

Canister, PickupScheduleCanister

49. Field: canRead

Description:

true if user has read permission

\section{Tables:}

PickupSchedulePermission, ScenarioPermission

\section{Field: canWrite}

\section{Description:}

true if user has write permission

\section{Tables:}

PickupSchedulePermission, ScenarioPermission

\section{Field: capitalCost \\ Description:}

acquisition cost to purchase (\$)

\section{Tables:}

Canister, Cask, VehicleType

\section{Field: carCost}

\section{Description:}

cost per car

\section{Tables:}

Cost 


\section{Field: caskID}

\section{Description:}

cask ID number (internal ID number assigned to an individual cask to facilitate tracking through shipments)

\section{Tables:}

ScenarioCaskMaintenance, TripCaskXref

\section{Field: caskMaintFacility}

\section{Description:}

site ID of cask maintenance facility

\section{Tables:}

Scenario

\section{Field: caskName}

\section{Description:}

cask name

\section{Tables:}

Cask

\section{Field: caskTypelD}

\section{Description:}

cask type ID number

\section{Tables:}

Cask, PickupScheduleCask, ScenarioCaskMaintenance, TripCaskXref

\section{Field: completed}

\section{Description:}

date job was completed

\section{Tables:}

Job

\section{Field: cost}

\section{Description:}

cost (\$)

\section{Tables:}

ScenarioCost, TripCost 
59. Field: costCategory

\section{Description:}

cost category (Capital, Maintenance, or Operations)

\section{Tables:}

ScenarioCost, TripCost

60. Field: costDetail

Description:

cost detail

\section{Tables:}

ScenarioCost, TripCost

\section{Field: costYear}

\section{Description:}

base year for cost inflation (set to current year to inflate past years' costs to current dollars)

\section{Tables:}

Scenario

\section{Field: craneOnsiteTime}

\section{Description:}

time crane is onsite (hours)

\section{Tables:}

SimulationOutput

\section{Field: craneOperationTime}

\section{Description:}

time crane is in use (hours)

\section{Tables:}

SimulationOutput

\section{Field: dailyCost}

\section{Description:}

cost per day

\section{Tables:}

Cost 
65. Field: decommissioningCost

Description:

cost to dispose of cask $(\$)$

\section{Tables:}

Cask

66. Field: defaultConsistSize

Description:

default consist size (can be overridden by consistSize field in the ScenarioSite table)

\section{Tables:}

Scenario

67. Field: derivation

Description:

source of data $(\mathrm{C}=\mathrm{CTA}$ network, $\mathrm{E}=$ estimate, $\mathrm{T}=\mathrm{TRAGIS})$

\section{Tables:}

SiteDistance

68. Field: description

Description:

cask description

Tables:

Cask

69. Field: description

Description:

problem description

Tables:

ScenarioErrorLog

\section{Field: description}

\section{Description:}

vehicle description

\section{Tables:}

VehicleType 


\section{Field: destination}

\section{Description:}

destination location (either ISF or MGR)

\section{Tables:}

PickupScheduleCanister, PickupScheduleCask, Trip

72. Field: detail Description:

cost description

\section{Tables:}

Cost

\section{Field: distance}

Description:

leg distance (miles)

\section{Tables:}

SiteDistance

\section{Field: emptyWeight}

\section{Description:}

empty cask weight (in pounds)

\section{Tables:}

Cask

\section{Field: endDate}

\section{Description:}

end date

\section{Tables:}

TripTime

\section{Field: endYear}

\section{Description:}

analysis end year

\section{Tables:}

Scenario 


\section{Field: equipmentDescription}

Description:

description

\section{Tables:}

Equipment

78. Field: equipmentID

Description:

equipment ID number (sequential number for the individual assets used for tracking)

\section{Tables:}

ScenarioAcquisition

79. Field: equipmentType

Description:

category of equipment (CASK or VEHICLE)

\section{Tables:}

ScenarioAcquisition

\section{Field: equipmentTypelD}

\section{Description:}

equipment type ID number (cask ID number for CASK assets, and vehicle ID number of VEHICLE assets)

\section{Tables:}

ScenarioAcquisition

\section{Field: fromMode}

\section{Description:}

mode from which the cask is transferred

\section{Tables:}

SimulationTransferTime

\section{Field: fromSitelD}

\section{Description:}

site ID number

\section{Tables:}

SiteDistance 


\section{Field: fuelType}

\section{Description:}

fuel type $(\mathrm{B}=\mathrm{BWR}, \mathrm{P}=\mathrm{PWR})$

\section{Tables:}

Canister

\section{Field: fuelType}

Description:

fuel type ( $\mathrm{B}=\mathrm{BWR}, \mathrm{P}=\mathrm{PWR}, \mathrm{H}=$ ?, $\mathrm{X}=$ ?)

\section{Tables:}

Cask

\section{Field: heavyHaulOnsiteTime \\ Description:}

time $\mathrm{HH}$ is onsite (hours)

\section{Tables:}

SimulationOutput

86. Field: heavyHaulOperationTime

\section{Description:}

time $\mathrm{HH}$ is in operation (hours)

\section{Tables:}

SimulationOutput

\section{Field: hourlyCost}

\section{Description:}

cost per hour

Tables:

Cost

\section{Field: includesShortline}

\section{Description:}

true if shortline rail is included in route

\section{Tables:}

SiteDistance 
89. Field: inflationRate

Description:

yearly inflation rate (as a percent, so e.g., $5.2 \%=5.2$ )

\section{Tables:}

Inflation

90. Field: isLoaded Description:

true if the cask is loaded

\section{Tables:}

SimulationTransferTime

91. Field: isOverpack

Description:

true if cask is an overpack

\section{Tables:}

Cask

\section{Field: latitude}

\section{Description:}

latitude (degrees)

\section{Tables:}

Site, SiteNode

\section{Field: leg}

\section{Description:}

leg number

\section{Tables:}

SiteDistance

\section{Field: legMode}

\section{Description:}

mode for leg $(\mathrm{H}=$ highway, $\mathrm{R}=$ rail, $\mathrm{W}=$ waterway $)$

\section{Tables:}

SiteDistance 


\section{Field: lifetime}

\section{Description:}

usable life of cask (years)

\section{Tables:}

Cask

96. Field: loadDate

Description:

date that schedule was loaded

\section{Tables:}

PickupSchedule

\section{Field: loadedWeight}

Description:

fully loaded cask weight (in pounds)

\section{Tables:}

Cask

\section{Field: loadTime}

\section{Description:}

time to load cask (hours)

\section{Tables:}

Cask

\section{Field: location}

\section{Description:}

location of fuel ( $\mathrm{P}=$ pool, $\mathrm{D}=$ dry storage)

\section{Tables:}

PickupScheduleCanister, PickupScheduleCask

\section{Field: longitude}

\section{Description:}

longitude (degrees)

\section{Tables:}

Site, SiteNode 


\section{Field: maintenanceDate}

\section{Description:}

date of maintenance

\section{Tables:}

ScenarioCaskMaintenance

\section{Field: maintenanceDate}

\section{Description:}

maintenance date

\section{Tables:}

ScenarioVehicleMaintenance

\section{Field: maintenanceType}

\section{Description:}

type of maintenance (standard or annual)

\section{Tables:}

ScenarioCaskMaintenance

\section{Field: maintenanceType}

\section{Description:}

type of maintenance (standard or mileage)

\section{Tables:}

ScenarioVehicleMaintenance

\section{Field: maxConsistSize}

\section{Description:}

maximum consist size (overwrites defaultConsistSize from the Scenario table)

\section{Tables:}

ScenarioSite

\section{Field: mileageCost}

\section{Description:}

cost per mile

\section{Tables:}

Cost 
107. Field: mileagelnspectionCost

Description:

mileage inspection cost $(\$)$

\section{Tables:}

VehicleType

\section{Field: mileagelnspectionTime} Description:

mileage inspection time (hours)

\section{Tables:}

VehicleType

109. Field: mileageSquaredCost

\section{Description:}

cost per mile squared

\section{Tables:}

Cost

\section{Field: minCost}

\section{Description:}

minimum cost

\section{Tables:}

Cost

\section{Field: mode}

\section{Description:}

overall mode of travel $(\mathrm{H}=$ highway, $\mathrm{R}=$ rail $)$

\section{Tables:}

SiteDistance

\section{Field: mode}

\section{Description:}

transportation mode $(\mathrm{H}=$ highway, $\mathrm{L}=$ local, $\mathrm{R}=$ rail $)$

\section{Tables:}

Cost 


\section{Field: mode}

Description:

usage mode $(\mathrm{R}=$ rail transportation, $\mathrm{T}=$ truck transportation, $\mathrm{S}=$ storage $)$

\section{Tables:}

Cask

\section{Field: monthlyCost}

Description:

cost per month

\section{Tables:}

Cost

\section{Field: name}

Description:

routing option name

\section{Tables:}

SiteTransportationOption

\section{Field: name}

Description:

run name

\section{Tables:}

Scenario

\section{Field: nodeType}

\section{Description:}

node type (road, water dock, rail, water rail, rail siding)

\section{Tables:}

SiteNode

\section{Field: numCasks}

\section{Description:}

consist size

\section{Tables:}

SimulationOutput 


\section{Field: numOfCanisters}

\section{Description:}

number of canisters to be picked up

\section{Tables:}

PickupScheduleCanister

\section{Field: numOfCasks}

\section{Description:}

number of casks to be picked up

\section{Tables:}

PickupScheduleCask

\section{Field: optionID}

\section{Description:}

routing option ID number

\section{Tables:}

SiteDistance, SiteNode, SiteTransportationOption

\section{Field: origin}

\section{Description:}

origin location (either reactor site ID number or ISF)

\section{Tables:}

Trip

\section{Field: origin}

\section{Description:}

pickup location (either reactor site ID number, or ISF)

\section{Tables:}

PickupScheduleCanister, PickupScheduleCask

\section{Field: originalSimScenariolD}

\section{Description:}

$<$ unused $>$

\section{Tables:}

Site 
125. Field: otherCost

Description:

other cost

Tables:

Cost

\section{Field: otherDescription} Description:

other cost description

Tables:

Cost

127. Field: owner

Description:

owner (user who created the run)

\section{Tables:}

Scenario

\section{Field: owner}

\section{Description:}

owner (user who input schedule)

\section{Tables:}

PickupSchedule

\section{Field: problemID}

Description:

problem ID number (sequential ID for run)

\section{Tables:}

ScenarioErrorLog

\section{Field: queued}

\section{Description:}

date job was added to the queue

\section{Tables:}

Job 


\section{Field: railcarMaintFacility}

\section{Description:}

site ID of railcar maintenance facility

\section{Tables:}

Scenario

\section{Field: repositorySitelD}

\section{Description:}

site ID of repository

\section{Tables:}

Scenario

\section{Field: runCompleted}

\section{Description:}

true if the run has completed

\section{Tables:}

Scenario

\section{Field: runDate}

\section{Description:}

date of the last run

\section{Tables:}

Scenario

\section{Field: runID}

\section{Description:}

run ID number

\section{Tables:}

Job, Scenario, ScenarioAcquisition, ScenarioCaskMaintenance, ScenarioCost, ScenarioErrorLog, ScenarioISF, ScenarioPermission, ScenarioSite, ScenarioVehicleMaintenance, Trip, TripCaskXref, TripCost, TripTime, TripVehicleXref

\section{Field: runMode}

\section{Description:}

run mode for scheduling program $(\mathrm{Q}=$ quick, $\mathrm{O}=$ optimized $)$

\section{Tables:}

Scenario 


\section{Field: runRequested}

\section{Description:}

true if a run request has been made

\section{Tables:}

Scenario

\section{Field: scheduleID}

Description:

schedule ID number

\section{Tables:}

PickupSchedule, PickupScheduleCanister, PickupScheduleCask, PickupSchedulePermission, Scenario

\section{Field: scheduleName}

\section{Description:}

schedule name

\section{Tables:}

PickupSchedule

\section{Field: securityTime}

\section{Description:}

time before departure that security must be present (hours)

\section{Tables:}

SimulationOutput

\section{Field: simScenariolD}

\section{Description:}

simulation scenario ID number

\section{Tables:}

SimScenario, SimulationOutput, Site

\section{Field: simScenarioName}

\section{Description:}

simulation scenario name

\section{Tables:}

SimScenario 
143. Field: sitelD

Description:

site ID number

\section{Tables:}

ScenarioISF, ScenarioSite, Site, SiteNickname, SiteNode, SiteTransportationOption

\section{Field: siteName}

Description:

site name

Tables:

Site

\section{Field: siteNickName}

Description:

site nickname

\section{Tables:}

SiteNickname

\section{Field: speed}

\section{Description:}

speed of equipment (mph)

\section{Tables:}

Equipment

\section{Field: standardlnspectionCost}

\section{Description:}

inspection cost after each trip (\$)

\section{Tables:}

Cask, VehicleType

\section{Field: standardlnspectionTime}

\section{Description:}

inspection time after each trip (hours)

\section{Tables:}

Cask, VehicleType 


\section{Field: startDate}

Description:

start date

\section{Tables:}

TripTime

150. Field: started

Description:

date job was started

\section{Tables:}

Job

\section{Field: startYear}

Description:

analysis start year

\section{Tables:}

Scenario

\section{Field: stateList}

\section{Description:}

FIPS codes of states traversed

\section{Tables:}

SiteDistance

\section{Field: status}

\section{Description:}

job status (queued, started, finished)

\section{Tables:}

Job

\section{Field: toMode}

\section{Description:}

mode to which the cask is transferred

\section{Tables:}

SimulationTransferTime 


\title{
155. Field: toSiteID
}

\section{Description:}

site ID number

\section{Tables:}

SiteDistance

\section{Field: totalTime} Description:

total time for loading operation (minus loading fuel into casks and/or canisters) (hours)

\section{Tables:}

SimulationOutput

\section{Field: trailerMaintFacility}

\section{Description:}

site ID of truck trailer maintenance facility

\section{Tables:}

Scenario

\section{Field: transferTime}

\section{Description:}

transfer time (hours)

\section{Tables:}

SimulationTransferTime

\section{Field: transportationCaskTypeID}

\section{Description:}

cask type ID number of transportation overpack

\section{Tables:}

\author{
Canister
}

\section{Field: travelTime}

\section{Description:}

leg travel time (hours)

\section{Tables:}

SiteDistance 


\section{Field: tripCost}

\section{Description:}

cost per trip

\section{Tables:}

Cost

\section{Field: tripNumber}

\section{Description:}

trip number (internal)

\section{Tables:}

Trip, TripCaskXref, TripCost, TripTime, TripVehicleXref

163. Field: unloadTime

\section{Description:}

time to unload cask (hours)

\section{Tables:}

Cask

\section{Field: userName}

\section{Description:}

user name

\section{Tables:}

PickupSchedulePermission, ScenarioPermission, TSLUser

\section{Field: userName}

\section{Description:}

user who added job to queue

\section{Tables:}

Job

\section{Field: vehiclelD}

\section{Description:}

vehicle ID number (internal ID number assigned to an individual vehicle to facilitate tracking through shipments)

\section{Tables:}

ScenarioVehicleMaintenance, TripVehicleXref 
167. Field: vehicleType

Description:

vehicle type (R-Rail, T-Truck)

\section{Tables:}

VehicleType

168.Field: vehicleTypeID

Description:

vehicle type ID number

\section{Tables:}

ScenarioVehicleMaintenance, TripVehicleXref

169. Field: vehicleTypeID

Description:

vehilce type ID number

Tables:

VehicleType

170. Field: weightCost

Description:

cost per ton

Tables:

Cost

\section{Field: weightMileCost}

Description:

cost per ton mile

Tables:

Cost

\section{Field: weightMileSquaredCost}

Description:

cost per ton mile squared

\section{Tables:}

Cost 
173. Field: year

\section{Description:}

calendar year

\section{Tables:}

Inflation, PickupScheduleCanister, PickupScheduleCask

\section{Field: year}

Description:

calendar year of cost (used in inflation calculations)

\section{Tables:}

Cost 